\title{
Long-Term Survival, Morbidity, Social Functioning and Risk of Disability in Patients with a Herpes Simplex Virus Type I or Type 2 Central Nervous System Infection, Denmark, 2000-20I6
}

This article was published in the following Dove Press journal: Clinical Epidemiology

\begin{abstract}
Ann-Brit E Hansen, (iD) ${ }^{1-3}$
Hanne T Vestergaard, ${ }^{4}$ Ram B Dessau, (ID ${ }^{5}$ Jacob Bodilsen, (iD) ${ }^{6}$ Nanna S Andersen, (iD) Lars H Omland, (iD) Claus B Christiansen, ${ }^{8}$ Svend Ellermann-Eriksen, ${ }^{9}$ Lene Nielsen, ${ }^{10}$ Thomas Benfield, (iD) ${ }^{2,3}$

Henrik T Sørensen, (iD) 11

Christian $\varnothing$ Andersen, ${ }^{12}$ Anne-Mette Lebech, ${ }^{1,3}$ Niels Obel ${ }^{1,3}$

'Department of Infectious Diseases, Copenhagen University Hospital, Rigshospitalet, Copenhagen, Denmark; ' 2 Department of Infectious Diseases, Amager Hvidovre Hospital, University of Copenhagen, Hvidovre, Denmark; ${ }^{3}$ Department of Clinical Medicine, University of Copenhagen, Copenhagen, Denmark; ${ }^{4}$ Department of Virus \& Microbiological Special Diagnostics, Statens Serum Institute, Copenhagen, Denmark; ${ }^{5}$ Department of Clinical Microbiology, Slagelse Hospital, Slagelse, Denmark; ${ }^{6}$ Departments of Infectious Diseases and Clinical Microbiology, Aalborg University Hospital, Aalborg, Denmark; ${ }^{7}$ Department of Clinical Microbiology, Odense University Hospital, Odense, Denmark; ${ }^{8}$ Department of Clinical Microbiology,

Copenhagen University Hospital, Rigshospitalet, Copenhagen, Denmark; ${ }^{9}$ Department of Clinical Microbiology, Aarhus University Hospital, Aarhus, Denmark; ${ }^{10}$ Department of Clinical Microbiology, Herlev and Gentofte Hospital, University of Copenhagen, Herlev, Denmark;

"'Department of Clinical Epidemiology, Aarhus University Hospital, Aarhus, Denmark;

${ }^{12}$ Department of Clinical Microbiology, Amager Hvidovre University Hospital, University of Copenhagen, Hvidovre, Denmark
\end{abstract}

Correspondence: Ann-Brit E Hansen Department of Infectious Diseases, Copenhagen University Hospital, Rigshospitalet, Blegdamsvej 9,

Copenhagen $\varnothing$ DK-2100, Denmark Tel +4530299306

Email ann-brit.eg.hansen.02@regionh.dk
Background: The long-term prognosis following herpes simplex virus (HSV) central nervous system (CNS) infection is still debated.

Patients and Methods: We examined outcomes in all Danish residents who, during 2000-2016, tested PCR positive for HSV-1 $(n=208)$ or HSV-2 $(n=283)$ in the cerebrospinal fluid, compared to comparison cohorts from the general population ( $n=2080$ and $n=2830$ ).

Results: One-year mortality was increased among HSV-1 patients (difference 19.3\%; 95\% CI: $13.6 \%$ to $25.0 \%$ ) and HSV-2 patients (difference $5.3 \%$; $95 \%$ CI: $2.5 \%$ to $8.1 \%$ ), but thereafter mortality was not increased. After exclusion of persons diagnosed with cancer prior to study inclusion, one-year mortality difference for HSV-2 patients was $1.7 \%(-0.1 \%$ to $3.5 \%$ ). After five years, HSV-1 patients had lower employment (difference $-19.8 \%$; $95 \%$ CI: $-34.7 \%$ to $-4.8 \%$ ) and higher disability pension rates (difference $22.2 \%$; $95 \%$ CI: $8.4 \%$ to $36.0 \%$ ) than the comparison cohort, but similar number of inpatient days, outpatient visits, and sick leave. HSV-2 patients had employment and disability pension rates comparable to the comparison cohort, but more inpatient days (difference $1.5 /$ year; $95 \% \mathrm{CI}$ : -0.2 to 3.2 ), outpatient visits (difference 1.3/year; 95\% CI: 0.3 to 3.2), and sick leave days (difference 9.1/ year; $95 \%$ CI: 7.9 to 10.4$)$.

Conclusion: HSV-1 and HSV-2 CNS infections differ substantially with respect to prognosis. HSV-1 CNS infection is followed by increased short-term mortality and long-term risk of disability. HSV-2 CNS infection has no substantial impact on mortality or working capability but is associated with increased morbidity.

Keywords: herpes simplex virus encephalitis, herpes simplex virus meningitis, prognosis, outcome, cohort study

\section{Introduction}

Herpes simplex viruses (HSVs) are well-known pathogens of viral central nervous system (CNS) infections ${ }^{1,2}$ and can manifest as both meningitis and encephalitis. HSV is the most common cause of sporadic fatal encephalitis globally, ${ }^{1,3}$ and the majority of cases (up to 90\%) are caused by HSV type 1 (HSV-1)., ${ }^{4,5}$ Antiviral therapy of HSV encephalitis has improved the prognosis, ${ }^{6}$ but mortality is still $10-20 \%,{ }^{4}$ and a recent study of narrative interviews emphasized the magnitude of challenges and socioeconomic consequences still faced by these patients years after being treated for acute HSV encephalitis. ${ }^{7}$ 
The main clinical manifestation of HSV type 2 (HSV2) CNS infection is meningitis. ${ }^{8}$ Some, but not all studies, have shown an increased risk of neurological morbidity after HSV-2 meningitis, ${ }^{8-10}$ and a recent UK-study found that patients with HSV-2 meningitis had a lower quality of life than healthy controls at one-year follow-up. ${ }^{2}$

Studies on long-term outcomes of HSV CNS infections are scarce and often hampered by small or selected study populations restricted to patients with severe HSV encephalitis, patients admitted to referral centers, or patients from a single center, and by lack of adequate comparison cohorts. $^{5,6,10,11}$ Follow-up rarely exceeds one year, ${ }^{12}$ and no studies have included complete national data on all HSV-1 and HSV-2 CNS infections. ${ }^{4,13-15}$ Information on the long-term prognosis following an episode of HSV CNS infection is essential for improving of clinical decision making and planning long-term patient management and will be of great utility to patients and health care providers alike. ${ }^{16}$

We used a nationwide population-based cohort design described in previous studies, ${ }^{17,18}$ to examine long-term survival, morbidity, social functioning and risk of disability among patients with HSV-1 and HSV-2 CNS infections, compared to age- and sex-matched comparison cohorts drawn from the general population.

\section{Patients and Methods}

\section{Setting}

On December 31, 2016, Denmark had a population of 5.7 million. Tax-supported health care is provided free of charge to all Danish residents. ${ }^{19}$

\section{Data Sources}

We used a nationwide population-based cohort design, described in previous studies. ${ }^{17,18}$ The unique 10-digit personal identification number, assigned to all Danish residents at birth or upon immigration, was used to track individuals in the Danish national health and administrative registries. Data on positive polymerase chain reaction (PCR) tests for HSV-1 and HSV-2 infections in the cerebrospinal fluid (CSF) were obtained from all Danish microbiology laboratories performing the test during the period January 1, 2000 to March 1, 2016. Additional data were obtained from the Danish Civil Registration System (CRS), the Danish National Patient Registry (DNPR), the Danish Cancer Registry, the Employment Classification Module, the Danish Educational Attainment Registry as well as housing statistics from Statistics Denmark (see Supplementary Appendices 1-4). ${ }^{19}$

\section{Study Population}

HSV Patient Cohorts

The patient cohorts included Danish residents with a positive PCR test for HSV-1 or HSV-2 in the CSF during the period January 1, 2000-March 1, 2016. We defined the date of the first positive HSV PCR test in the CSF as the date of study inclusion.

\section{Population Comparison Cohorts}

For each HSV patient, we used the CRS to identify all Danish residents who had not tested PCR-positive for HSV-1 or HSV-2 in the CSF and with the same sex and date of birth as the patient. ${ }^{20}$ From this population, we extracted 10 individuals at random for each patient and constructed a comparison cohort for the HSV-1 cohort and a comparison cohort for the HVS-2 cohort. Individuals in the comparison cohorts were assigned the same date of study inclusion as the HSV-1 or HSV-2 patients to whom they were matched.

\section{Outcomes}

Outcomes were categorized into the following categories: (1) all-cause mortality and risk of cancer, (2) neuropsychiatric sequelae (dementia and epilepsy), (3) health care utilization as a measure of morbidity (hospitalizations, outpatient visits, visits to a department of neurology), (4) outcomes that might reflect impaired social functioning or disability (unemployment, disability pension, sick leave, nursing home residency, marriage and divorce rates), and (5) unfavorable outcome defined as a combined endpoint of death and measures of severe disability (receipt of a disability pension, nursing home residency, dementia).

\section{Statistical Analyses}

We calculated time from date of study inclusion to 1 March 2016, death, emigration, loss to follow up, or event of interest, whichever came first. Mortality rate ratios (MRRs) and incidence rate ratios (IRRs) were calculated as measures of relative risk.

For each person in the HSV-1 and HSV-2 cohorts and their corresponding comparison cohorts, we ascertained the yearly number of hospital inpatient days, outpatient hospital visits, proportion visiting a neurology department, proportion having a hospital contact associated with a diagnosis of epilepsy, employment status, sick leave days, receipt of 
a disability pension, marital status, and residency in a nursing home. Ascertainment started six years prior to the date of study inclusion, birth, immigration, or start-up date of the registry that recorded the outcome of interest. Data collection continued up until the earliest of the following events: six years after study inclusion, March 1, 2016 , death, emigration, or loss to follow-up. For each outcome, we calculated differences between the patient and comparison cohorts with $95 \%$ CIs. Analyses of sick leave, employment and receipt of a disability pension were restricted to individuals aged 20-60 years at study inclusion. In addition, analysis of sick leave only included individuals registered as employed. To account for potential differences in comorbidity among study participants at the time of study inclusion, we calculated the fraction of individuals with a Charlson Comorbidity Index (CCI) score $>0$ at study inclusion. ${ }^{21,22}$ We performed supplementary sensitivity analyses of mortality, time to unfavorable outcome, annual number of hospital inpatient days and outpatient visits, in which we excluded individuals with a diagnosis of cancer prior to the date of study inclusion.

SPSS Statistics, version 24 (SPSS, Inc., Chicago, Illinois) and STATA version 14 were used for analysis.

\section{Approvals and Ethics}

In Denmark, research based on registry data without direct patient interaction does not require scientific ethical approval. The study was approved by the Danish Data Protection Agency and the National Board of Health (RH-2015-285, I-Suite no.: 04297).

\section{Results}

During the study period, we identified 208 persons with a positive PCR test for HSV-1 and 283 with a positive test for HSV-2 in the CSF and included 2080 and 2830 persons in the matched HSV-1 and HSV-2 comparison cohorts. The HSV-1 group was older than the HSV-2 group (median age 60 years versus 38 years) and included fewer women (54\% versus $71 \%$ ). Both HSV-1 and HSV-2 patients had slightly higher CCI scores and prevalence of cancer prior to study inclusion than members of the comparison cohorts (Table 1).

\section{Mortality and Risk of Cancer}

During the observation period, 69 (33\%) HSV-1 patients and $21(7 \%)$ HSV-2 patients died compared with 288 (13\%) and $77(3 \%)$ deaths in the corresponding comparison cohorts (Table 1). Risk of death was substantially increased in the first year after diagnosis of HSV CNS infection; MRR was
10.9 (95\% CI: 7.3 to 16.4$)$ for HSV-1 patients and $8.4(95 \%$ CI: 4.4 to 16.0) for HSV-2 patients. One-year absolute excess mortality was $19.3 \%$ (95\% CI: $13.6 \%$ to $25.0 \%)$ in the HSV-1 group and $5.3 \%$ (95\% CI: $2.5 \%$ to $8.1 \%$ ) in the HSV-2 group. Eleven of 17 patients, who died within the first year after diagnosis of an HSV-2 CNS infection, had been diagnosed with cancer before the HSV-2 diagnosis. Exclusion of persons diagnosed with cancer before study inclusion changed the oneyear risk difference estimate only marginally for the HSV-1 group but reduced the estimate for HSV-2 patients to $1.7 \%$ (95\% CI: $-0.1 \%$ to $3.5 \%$ ). After one year, neither mortality nor risk of a new cancer diagnosis were substantially increased among HSV-1 or HSV-2 patients (Table 2, Figure 1).

\section{Neuropsychiatric Sequelae}

The risk of a dementia diagnosis was increased in the HSV1 cohort the first years after HSV-1 CNS infection with a five-year risk of $4.6 \%$ (95\% CI: $1.2 \%$ to $7.9 \%$ ) corresponding to a five-year risk difference of $4.0 \%$ (95\% CI: $0.6 \%$ to $7.4 \%$ ) (Supp. Figure 1). In contrast, no individuals in the HSV-2 cohort were diagnosed with dementia. HSV-1 patients had more hospital contacts with a diagnosis of epilepsy from the year of their HSV-1 CNS infection and throughout follow up. Five years after study inclusion, the risk difference was $7.5 \%$ (95\% CI: $1.9 \%$ to $13.1 \%$ ). During the year of diagnosis, HSV-2 patients had more hospital contacts associated with a diagnosis of epilepsy than members of the comparison cohort but thereafter the risk waned and approached that observed in the comparison cohort (Table 3, Supp. Figure 2).

\section{Health Care Utilization}

During the year of HSV diagnosis, days of hospitalization and outpatient visits were considerably higher in both patient cohorts than in the comparison cohorts. Thereafter, use of hospital services decreased but remained somewhat higher in the HSV-2 cohort than in the comparison cohort (Table 3, Supp. Figure 3). Of note, HSV-2 patients also had more days of hospitalization and more outpatient visits in the years prior to diagnosis. In a sensitivity analysis excluding individuals with a cancer diagnosis prior to study inclusion, HSV-2 patients still showed increased use of hospital services before as well as after HSV-2 diagnosis.

After diagnosis of an HSV CNS infection, the proportion visiting a neurology department annually was increased among both HSV-1 and HSV-2 patients. After five years, the risk differences were 18.3\% (95\% CI: 9.9\% 
Table I Characteristics of Patients with HSV-I and HSV-2 in Cerebrospinal Fluid, and Their Corresponding Comparison Cohorts, Denmark, 2000-2016

\begin{tabular}{|c|c|c|c|c|}
\hline Characteristics & $\begin{array}{l}\text { HSV-I } \\
\text { Cohort }\end{array}$ & $\begin{array}{l}\text { Population Comparison } \\
\text { Cohort HSV-I }\end{array}$ & $\begin{array}{l}\text { HSV-2 } \\
\text { Cohort }\end{array}$ & $\begin{array}{l}\text { Population Comparison } \\
\text { Cohort HSV-2 }\end{array}$ \\
\hline Total & 208 & 2080 & 283 & 2830 \\
\hline Female sex - no. (\%) & $112(54)$ & $1120(54)$ & $20 I(7 I)$ & $2010(71)$ \\
\hline $\begin{array}{l}\text { Median age at study inclusion - years } \\
\text { (interquartile range) }\end{array}$ & $60(4 I-70)$ & $60(4 I-70)$ & $38(28-48)$ & $38(28-48)$ \\
\hline Age below 16 years at study inclusion (\%) & $16(8)$ & $160(8)$ & $7(2)$ & $70(2)$ \\
\hline $\begin{array}{l}\text { Age between } 20-60 \text { years at study inclusion } \\
\text { (\%) }\end{array}$ & $90(43)$ & $900(43)$ & $247(87)$ & $2470(87)$ \\
\hline Born in Denmark - no. (\%) & $197(95)$ & $1922(92)$ & $266(94)$ & $2486(88)$ \\
\hline High school education (\%) & III (53) & $1116(54)$ & $188(66)$ & $1893(67)$ \\
\hline $\begin{array}{l}\text { Charlson Comorbidity Index score >0 - no. } \\
(\%)^{\top, \$}\end{array}$ & $73(35)$ & $547(26)$ & $57(20)$ & $366(13)$ \\
\hline Cancer, total - no. $(\%)^{\$}$ & $29(14)$ & $192(9)$ & $21(7)$ & $108(4)$ \\
\hline $\begin{array}{l}\text { Median observation time }- \text { years } \\
\text { (interquartile range) }\end{array}$ & $\begin{array}{l}3.7 \\
(0.7-9.1)\end{array}$ & $6.2(2.5-10.1)$ & $\begin{array}{l}5.2 \\
(2.2-9.9)\end{array}$ & $5.5(2.7-10.5)$ \\
\hline Total observation time, years & $107 \mid$ & $|3,43|$ & $167 \mid$ & 18,114 \\
\hline Died - no. $(\%)^{\#}$ & $69(33)$ & $288(14)$ & $21(7)$ & $77(3)$ \\
\hline Diagnosed with cancer - no. (\%) & $12(6)$ & $161(8)$ & $7(2)$ & $83(3)$ \\
\hline Diagnosed with dementia - no. $(\%)^{\#}$ & $7(3)$ & $21(1)$ & $0(0)$ & $7(0)$ \\
\hline Emigrated - no. (\%) & $3(1)$ & $26(1)$ & II (4) & $43(2)$ \\
\hline Lost to follow up - no. $(\%)^{\#}$ & $0(0)$ & $\mathrm{I}(0)$ & $0(0)$ & $5(1)$ \\
\hline
\end{tabular}

Notes: "The Charlson Comorbidity Index $(\mathrm{CCl})$ score derived from diagnoses recorded in the Danish National Patient Registry at study inclusion. For simplicity, we dichotomized scores $(\mathrm{CCl}$ score $=0$ and $\mathrm{CCl}$ score $>0)$. ${ }^{\$}$ Diagnosed before study inclusion, ${ }^{\#}$ After study inclusion.

Abbreviations: HSV-I, herpes simplex virus type I; HSV-2, herpes simplex virus type 2.

to $26.7 \%$ ) for $\mathrm{HSV}-1$ patients and $5.5 \%$ (95\% CI: $1.2 \%$ to 9.8\%) for HSV-2 patients (Table 3, Supp. Figure 4).

\section{Unemployment, Sick Leave and Receipt of Disability Pension, Nursing Home Residency, Marriage and Divorce Rates}

The employment rate for the HSV-1 cohort was slightly lower than in the comparison cohort prior to diagnosis of HSV-1 CNS infection, but thereafter the employment rates decreased substantially. In contrast, the HSV-2 cohort did not have decreased employment rates before or after diagnosis (Table 3, Supp. Figure 5).

Among HSV-1 patients, the number of sick leave days was higher than for members of the comparison cohort during the year of diagnosis and the year thereafter, but two years after diagnosis the number of sick leave days was similar to that in the comparison cohort (Table 3, Supp. Figure 5). The number of sick leave days in the HSV-2 cohort was higher than for the comparison cohort in the year of study inclusion and throughout follow-up.

Before study inclusion, the proportion on disability pensions was slightly higher in the HSV-1 cohort than in the comparison cohort. After the HSV-1 diagnosis, the proportion on disability pension increased considerably. In contrast, the proportion on disability pension did not differ greatly between the HSV-2 cohort and the comparison cohort (Table 3, Figure 2).

More patients resided at a nursing home after diagnosis of HSV CNS infections than members of the comparison cohorts. One-year risk differences were $17.3 \%$ (95\% CI: $11.8 \%$ to $22.8 \%$ ) for HSV-1 patients and $1.8 \%$ (95\% CI: $0.0 \%$ to $3.6 \%$ ) for HSV-2 patients.

The proportion of HSV-1 patients who were married was almost identical to that observed in the comparison cohort, while the HSV-2 patients were less likely to be married than the comparison cohort (Table 3, Supp. Figure 6). The proportion of HSV-1 patients who were divorced seemed to increase slightly after diagnosis of their HSV CNS infection. In contrast, the proportion of divorce in the HSV-2 cohort was higher than in the comparison cohort before as well as after diagnosis of their HSV-2 CNS infection (Table 3, Supp. Figure 6).

\section{Unfavorable Outcome}

To examine the overall risk of an unfavorable outcome, we computed time to an adverse outcome defined as the 
Table 2 Mortality Rate Ratios (MRRs) and Incidence Rate Ratios (IRRs) for Cancer and Dementia Diagnoses in Patients with HSV-I and HSV-2 in Cerebrospinal Fluid Compared with Members of the Matched Comparison Cohorts

\begin{tabular}{|l|l|l|l|l|}
\hline & \multicolumn{2}{l|}{$\begin{array}{l}\text { HSV-I Patients versus Comparison } \\
\text { Cohort Members }\end{array}$} & \multicolumn{2}{l|}{$\begin{array}{l}\text { HSV-2 Patients versus Comparison } \\
\text { Cohort Members }\end{array}$} \\
\hline Mortality & MRR & $95 \% \mathrm{Cl}$ & MRR & $95 \% \mathrm{Cl}$ \\
\hline $\begin{array}{l}\text { Mortality in first year of follow-up } \\
\text { Mortality from the second year and onwards }\end{array}$ & $\begin{array}{l}10.9 \\
1.3\end{array}$ & $\begin{array}{l}7.3,16.4 \\
0.8,1.9\end{array}$ & $\begin{array}{l}8.4 \\
0.8\end{array}$ & $\begin{array}{l}4.4,16.0 \\
0.3,2.2\end{array}$ \\
\hline Morbidity & IRR & $\mathbf{9 5 \%} \mathbf{C l}$ & $\mathbf{I R R}$ & $\mathbf{9 5 \%} \mathbf{C l}$ \\
\hline Cancer & 0.9 & $0.5,1.7$ & 0.9 & $0.4,2.0$ \\
\hline
\end{tabular}

Abbreviations: HSV-I, herpes simplex virus type I; HSV-2, herpes simplex virus type 2; Cl, confidence interval.

composite endpoint of death, receipt of disability pension, dementia diagnosis, or nursing home residency. The five-year risk of an unfavorable outcome was $48 \%$ (95\% CI: $40 \%$ to

A

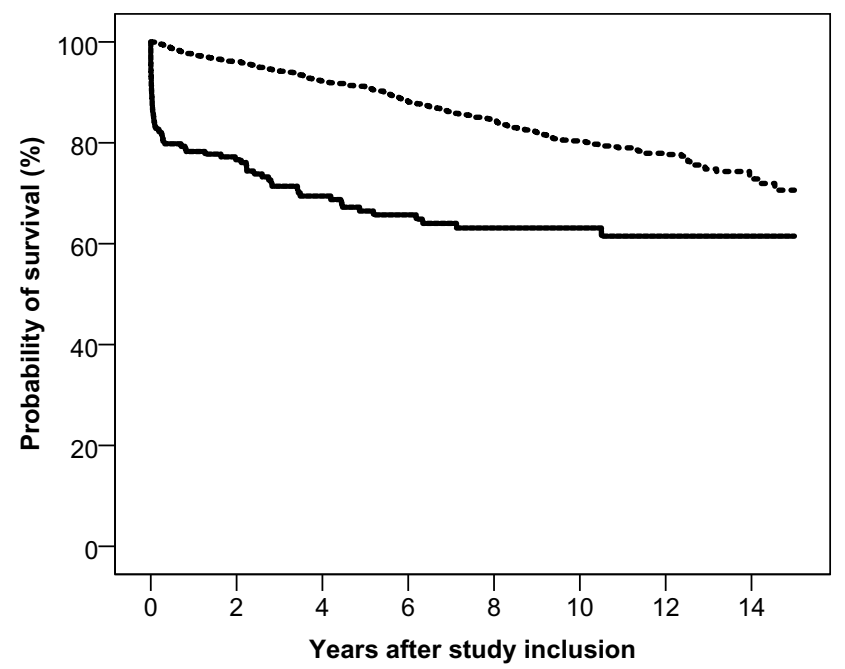

$55 \%$ ) in the HSV-1 cohort, corresponding to a risk difference of $36 \%$ (95\% CI: $28 \%$ to $44 \%$ ) relative to the comparison cohort. HSV-2 patients had an increased five-year risk of an

B

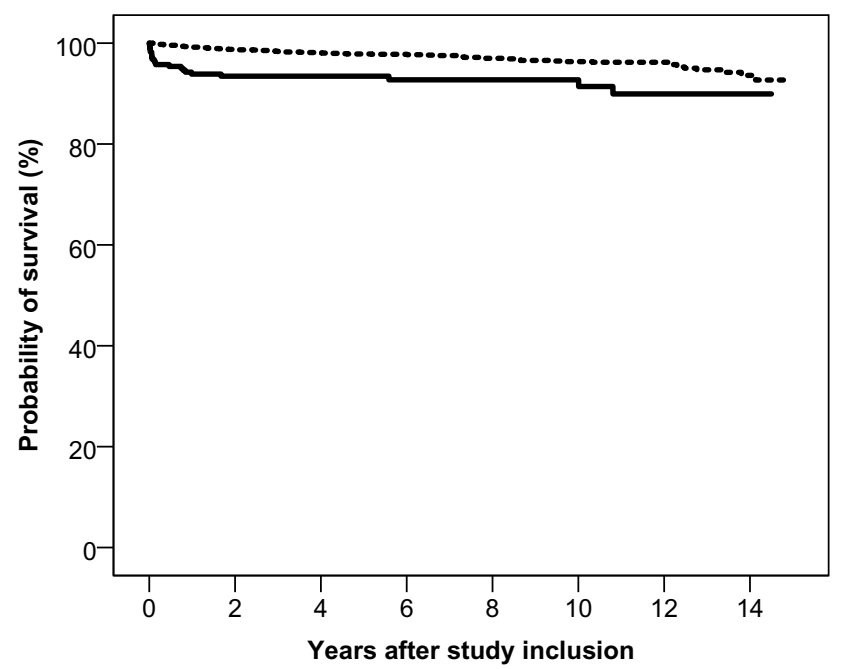

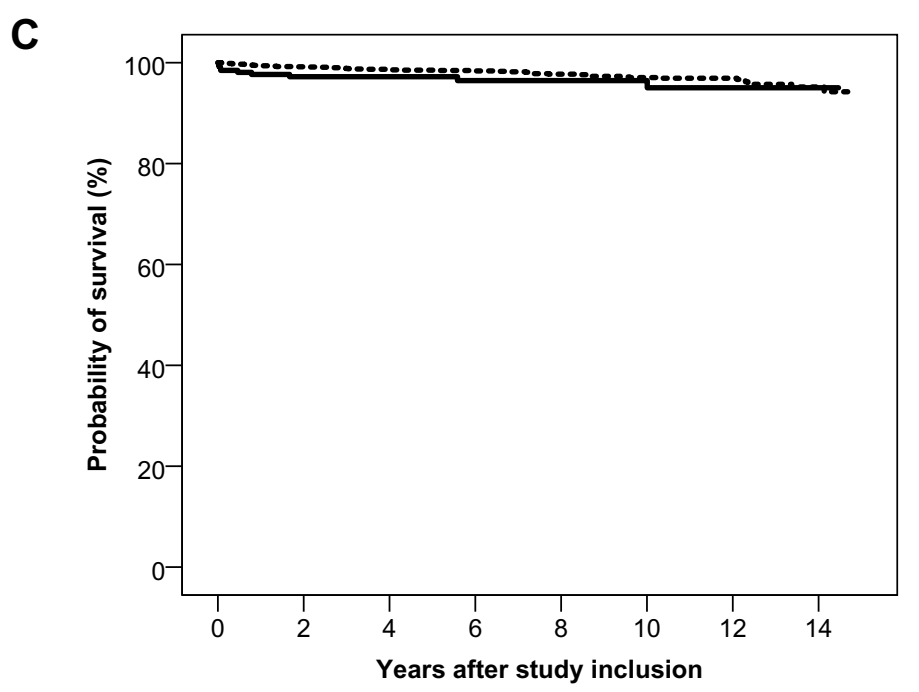

Figure I Survival in persons with herpes simplex virus (HSV) type I and type 2 central nervous system (CNS) infection (full lines) and in members of the comparison cohorts (broken lines). (A) Survival after HSV-I CNS infection. (B) Survival after HSV-2 CNS infection. (C) Survival after HSV-2 CNS infection in persons without a prior diagnosis of cancer. 
Table 3 Hospitalization Inpatient Days, Number of Outpatient Visits, Proportion Visiting a Department of Neurology, Proportion with a Hospital Visit Associated with Epilepsy, Employment Status, Sick Leave, Proportion Receiving Disability Pension, and Marital Status Among Patients with HSV-I or HSV-2 in Cerebrospinal Fluid and Members of the Comparison Cohorts

\begin{tabular}{|c|c|c|c|c|c|c|c|c|}
\hline $\begin{array}{l}\text { Years from } \\
\text { Study } \\
\text { Inclusion }\end{array}$ & $\begin{array}{l}\text { Patients } \\
\text { with HSV- } \\
\text { I }\end{array}$ & $\begin{array}{l}\text { Comparison } \\
\text { Cohort }\end{array}$ & Difference & $\begin{array}{l}95 \% \mathrm{Cl} \text { of } \\
\text { Difference }\end{array}$ & $\begin{array}{l}\text { Patients } \\
\text { with HSV- } \\
2\end{array}$ & $\begin{array}{l}\text { Comparison } \\
\text { Cohort }\end{array}$ & Difference & $\begin{array}{l}95 \% \mathrm{Cl} \text { of } \\
\text { Difference }\end{array}$ \\
\hline \multicolumn{9}{|c|}{ Hospitalization (mean inpatient days/year) } \\
\hline-2 & 1.5 & 1.0 & 0.5 & $-0.6,1.6$ & 1.3 & 0.5 & 0.9 & $-0.3,2.0$ \\
\hline 0 & 55.6 & 1.6 & 54.1 & $47.3,60.9$ & 16.2 & 0.8 & 15.4 & $11.9,19.0$ \\
\hline I & 2.5 & 1.5 & I.I & $-0.3,2.5$ & 1.5 & 0.7 & 0.8 & $0.1,1.5$ \\
\hline 2 & 3.2 & 1.3 & 1.9 & $0.1,3.6$ & 1.6 & 0.6 & 1.0 & $-0.1,2.1$ \\
\hline 5 & 1.7 & 1.4 & 0.3 & $-1.5,2.0$ & 2.1 & 0.6 & 1.5 & $-0.2,3.2$ \\
\hline \multicolumn{9}{|c|}{ Outpatient visits (mean visits/year) } \\
\hline-2 & 2.5 & 1.8 & 0.6 & $-0.3,1.5$ & 2.5 & 1.4 & 1.2 & $-0.1,2.4$ \\
\hline 0 & 11.7 & 2.1 & 9.6 & $4.2,14.9$ & 5.0 & 1.5 & 3.5 & $2.4,4.6$ \\
\hline I & 3.2 & 2.2 & 1.0 & $-0.1,2.1$ & 3.0 & 1.7 & 1.4 & $0.1,2.6$ \\
\hline 2 & 2.1 & 2.4 & -0.3 & $-1.2,0.5$ & 3.2 & 1.7 & 1.5 & $-0.2,3.2$ \\
\hline 5 & 2.3 & 2.4 & -0.1 & $-I . I, 0.8$ & 3.2 & 1.9 & 1.3 & $0.3,3.2$ \\
\hline \multicolumn{9}{|c|}{ Proportion visiting a department of neurology (\%) } \\
\hline-2 & 3.0 & 2.3 & 0.7 & $-1.7,3.1$ & 1.8 & 1.5 & 0.3 & $-1.4,1.9$ \\
\hline 0 & 33.2 & 1.9 & 31.3 & $24.9,37.7$ & 15.2 & 2.1 & 13.1 & $8.9,17.3$ \\
\hline 1 & 34.8 & 2.0 & 32.8 & $25.3,40.4$ & 10.3 & 2.2 & 8.1 & $4.3,11.9$ \\
\hline 2 & 23.6 & 2.3 & 21.3 & I4.2, 28.4 & 6.3 & 2.1 & 4.2 & I.I, 7.4 \\
\hline 5 & 20.2 & 2.0 & 18.3 & $9.9,26.6$ & 7.5 & 2.2 & 5.5 & I.2, 9.8 \\
\hline \multicolumn{9}{|c|}{ Proportion with a hospital visit associated with a diagnosis of epilepsy } \\
\hline-2 & 0.5 & 0.2 & 0.2 & $-0.7,1.2$ & 0.0 & 0.3 & -0.3 & $-0.5,-0.1$ \\
\hline 0 & 17.8 & 0.1 & 17.6 & $12.4,22.8$ & 2.8 & 0.2 & 2.6 & $0.7,4.6$ \\
\hline I & 17.4 & 0.1 & 17.3 & $11.3,23.3$ & 0.8 & 0.3 & 0.5 & $-0.7,1.6$ \\
\hline 2 & 10.0 & 0.2 & 9.8 & $4.8,14.7$ & 0.9 & 0.3 & 0.6 & $-0.6,1.9$ \\
\hline 5 & 7.9 & 0.3 & 7.5 & $1.9,13.1$ & 0.7 & 0.1 & 0.6 & $-0.8,1.9$ \\
\hline \multicolumn{9}{|c|}{ Proportion employed (\%) } \\
\hline-2 & 77.0 & 85.1 & -8.0 & $-17.2,1.1$ & 85.9 & 82.3 & 3.6 & $-1.1,8.3$ \\
\hline 0 & 72.5 & 82.4 & -9.9 & $-20.0,0.3$ & 78.7 & 79.7 & -1.0 & $-6.8,4.9$ \\
\hline 1 & 54.4 & 74.9 & -20.5 & $-32.7,-8.2$ & 79.3 & 80.7 & -1.4 & $-7.6,4.9$ \\
\hline 2 & 59.4 & 77.0 & -17.6 & $-30.0,-5.2$ & 75.8 & 77.5 & -1.8 & $-8.8,5.2$ \\
\hline 5 & 52.2 & 72.0 & -19.8 & $-34.7,-4.8$ & 80.6 & 77.0 & 3.6 & $-4.6,11.9$ \\
\hline \multicolumn{9}{|c|}{ Days of sick leave/year $\$$} \\
\hline-2 & 10.6 & 17.5 & -6.9 & $-7.7,-6.0$ & 23.5 & 23.0 & 0.5 & $-0.2,1.2$ \\
\hline 0 & 134.4 & 20.2 & 114.2 & $111.0,117.4$ & 49.9 & 24.3 & 25.7 & $24.5,26.8$ \\
\hline 1 & 97.1 & 14.9 & 82.2 & $78.9,85.4$ & 39.3 & 24.5 & 14.8 & $13.7,15.9$ \\
\hline 2 & 25.0 & 14.2 & 10.9 & $9.1,12.6$ & 21.7 & 24.4 & -2.7 & $-3.6,-1.8$ \\
\hline 5 & 6.9 & 11.2 & -4.3 & $-5.5,-3.1$ & 28.6 & 19.5 & 9.1 & $7.9,10.4$ \\
\hline \multicolumn{9}{|c|}{ Proportion on disability pension $(\%)^{\#}$} \\
\hline-2 & 12.6 & 7.6 & 5.1 & $-2.1,12.3$ & 5.1 & 3.7 & 1.4 & $-1.5,4.3$ \\
\hline 0 & 15.0 & 9.3 & 5.8 & $-2.3,13.8$ & 6.8 & 4.6 & 2.2 & $-1.4,5.7$ \\
\hline I & 19.1 & 10.4 & 8.7 & $-0.9,18.3$ & 6.3 & 5.0 & 1.3 & $-2.5,5.0$ \\
\hline
\end{tabular}

(Continued) 
Table 3 (Continued).

\begin{tabular}{|c|c|c|c|c|c|c|c|c|}
\hline $\begin{array}{l}\text { Years from } \\
\text { Study } \\
\text { Inclusion }\end{array}$ & $\begin{array}{l}\text { Patients } \\
\text { with HSV- } \\
\text { I }\end{array}$ & $\begin{array}{l}\text { Comparison } \\
\text { Cohort }\end{array}$ & Difference & $\begin{array}{l}95 \% \mathrm{Cl} \text { of } \\
\text { Difference }\end{array}$ & $\begin{array}{l}\text { Patients } \\
\text { with HSV- } \\
2\end{array}$ & $\begin{array}{l}\text { Comparison } \\
\text { Cohort }\end{array}$ & Difference & $\begin{array}{l}95 \% \mathrm{Cl} \text { of } \\
\text { Difference }\end{array}$ \\
\hline 2 & 28.1 & $1 \mathrm{I} .4$ & 16.7 & $5.4,28.0$ & 9.6 & 6.0 & 3.6 & $-1.2,8.3$ \\
\hline 5 & 32.6 & 10.4 & 22.2 & $8.4,36.0$ & 7.1 & 8.3 & -1.1 & $-6.5,4.2$ \\
\hline \multicolumn{9}{|c|}{ Proportion married (\%)* } \\
\hline-2 & 73.0 & 70.1 & 2.9 & $-3.7,9.6$ & $4 I .1$ & 47.2 & -6.2 & $-12.4,0.1$ \\
\hline 0 & 73.0 & 70.7 & 2.3 & $-4.3,9.0$ & 42.3 & 50.7 & -8.4 & $-14.6,-2.1$ \\
\hline I & 75.2 & 70.3 & 4.9 & $-2.6,12.3$ & 38.9 & 53.1 & -14.2 & $-20.7,-7.7$ \\
\hline 2 & 75.0 & 70.5 & 4.5 & $-3.3,12.3$ & 38.9 & 54.4 & -15.5 & $-22,4,-8.6$ \\
\hline 5 & 69.2 & 70.7 & -1.4 & $-12.0,9.2$ & 42.6 & 57.7 & -15.2 & $-23.7,-6.7$ \\
\hline \multicolumn{9}{|c|}{ Proportion divorced (\%)* } \\
\hline-2 & 7.9 & 11.4 & -3.4 & $-7.6,0.7$ & 14.1 & 8.0 & 6.1 & I.7, 10.4 \\
\hline 0 & 9.5 & 11.9 & -2.3 & $-6.8,2.1$ & 16.1 & 8.7 & 7.4 & $2.8,11.9$ \\
\hline I & 10.6 & 12.6 & -1.9 & $-7.2,3.4$ & 19.2 & 9.3 & 10.0 & $4.8,15.1$ \\
\hline 2 & 12.5 & 13.3 & -0.8 & $-6.8,5.2$ & 19.0 & 9.4 & 9.6 & 4.I. 15.0 \\
\hline 5 & 14.1 & 13.6 & 0.5 & $-7.5,8.5$ & 23.4 & 11.2 & 12.2 & $5.0,19.4$ \\
\hline
\end{tabular}

Notes: ${ }^{\#}$ Includes only individuals aged 20-60 years at study inclusion. *Includes only individuals aged $>20$ years at study inclusion. ${ }^{\$}$ Includes only individuals aged $20-60$ years at study inclusion and registered as employed.

Abbreviations: HSV-I, herpes simplex virus type I; HSV-2, herpes simplex virus type 2; $\mathrm{Cl}$, confidence interval.

unfavorable outcome [risk difference 5\% (95\% CI: $1 \%$ to $9 \%$ )], relative to the comparison cohort (Figure 3). The risk in HSV-2 patients was driven mainly by increased mortality during the first year after diagnosis, and after exclusion of persons diagnosed with cancer prior to study inclusion, the risk difference was reduced to $2 \%(95 \% \mathrm{CI}$ : $-2 \%$ to $5 \%$ ).

\section{Discussion}

In this nationwide population-based cohort study, we examined long-term outcomes following HSV-1 and HSV-2 CNS infections. During the first year following a diagnosis of HSV-1 CNS infection, the mortality was high with a fatal outcome in one-fifth of patients, and after five years nearly half of the patients had died or experienced severe disability. HSV-2 patients also had increased mortality in the first year following diagnosis of CNSinfection, but this was mainly explained by the higher proportion of patients diagnosed with cancer before study inclusion, and HSV-2 patients did not have increased longterm risk of severe outcomes.

The study's nationwide population-based design, large size, and the complete and long-term follow-up of the study cohorts allowed more precise risk estimates and a better understanding of long-term outcomes in the years following discharge from the acute hospital setting. Access to national registries allowed identification of well-matched populationbased comparison cohorts, and these registries provided complete data on date of death and comprehensive data on morbidity, neurological sequelae, and social functioning. We included cases based on detection of HSV DNA in the CSF. PCR detection of HSV in the CSF has a sensitivity and specificity above $95 \%{ }^{23}$ and we consider it a strength that all cases were microbiologically confirmed. We did not use the diagnoses of meningitis or encephalitis in our analyses, as the accuracy of these diagnoses in the DNPR is low. ${ }^{24}$ However, HSV-1 CNS infection presents almost exclusively as encephalitis, ${ }^{3}$ while HSV-2 CNS infection presents mainly as meningitis, ${ }^{10}$ although up to $10-15 \%$ of patients with HSV-2 CNS infections do present as encephalitis or meningoencephalitis. ${ }^{5,8}$ We did not have access to clinical information such as neuropsychological evaluations or functional motor assessments in patients with an HSV CNS infection, but we were able to evaluate the level of social functioning regarding important aspects of life such as working capability, marital status and nursing home residence and to compare this functional status with that in the general population using prospectively collected registry data. We started ascertainment six years prior to the diagnosis of HSV 
A

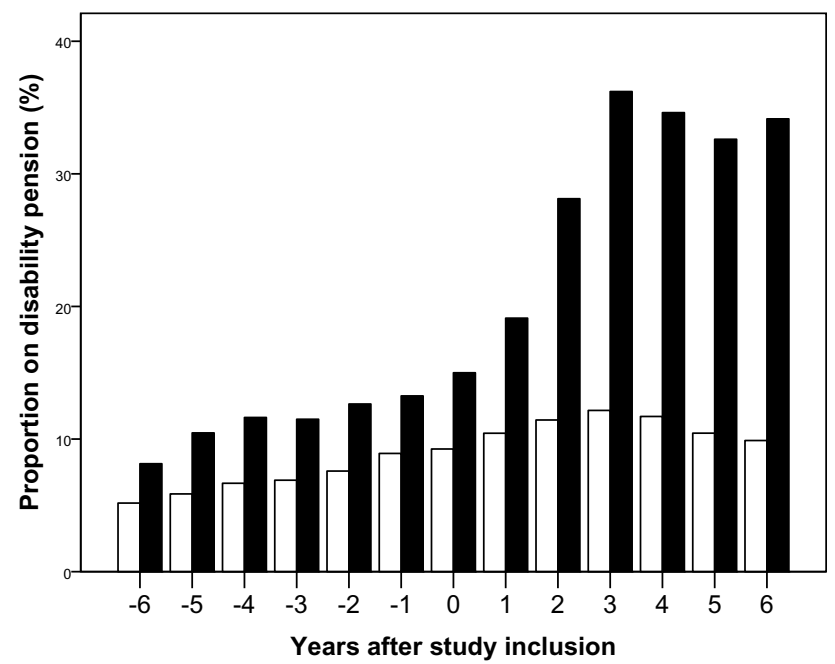

B

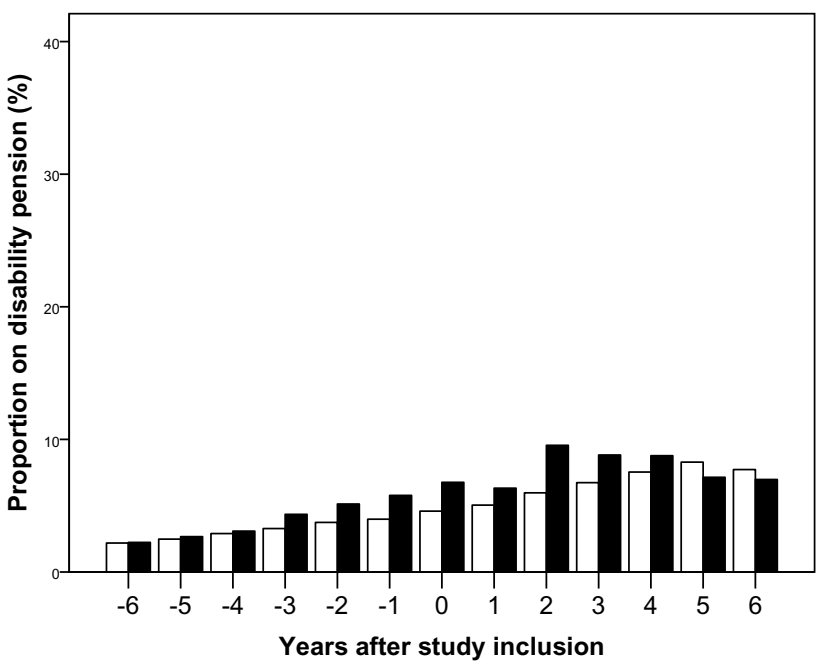

Figure 2 Proportion on disability pension* among patients with herpes simplex virus (HSV) type I and type 2 central nervous system infections (black) and members of the comparison cohorts (white) by years before and after study inclusion. (A) HSV-I CNS infection. (B) HSV-2 CNS infection.

Note: *Among individuals aged $20-60$ years at study inclusion.

CNS infection; thus, we were able to determine whether differences in outcomes between patient and population control cohorts were present prior to the diagnosis of HSV CNS infection.

We found very high one-year mortality following an episode of HSV-1 CNS infection with excess absolute mortality of $19 \%$. This finding is consistent with a previous Danish report of mortality among patients with HSV encephalitis, ${ }^{4}$ but higher than estimates presented in other population-based European studies, in which one-year mortality ranged from $10 \%$ to $14 \% .^{12-14}$ Importantly, our study provides new information about the serious long-term consequences of the diseases. After five years of follow-up, almost half of the HSV-1 group (47\%) had experienced either death or an unfavorable outcome. Further, the unemployment rate increased after the diagnosis of HSV-1 CNS infection, suggesting that working capability may also be affected among some patients who did not experience an unfavorable outcome. Some short-term studies have found neurological sequelae or disability in approximately $40-50 \%$ of the patients with HSV encephalitis. 5,6,14 Our study provides further documentation that the sequelae seem to translate into long-term disability.

A nationwide Swedish register-based study reported considerable morbidity after HSV encephalitis, including hospital readmission for epilepsy and neuropsychiatric conditions in $24 \%$ and $22 \%$ of survivors, respectively. ${ }^{12}$ We found that a large proportion of $\mathrm{HSV}-1$ patients had long-term neurological sequelae, including epilepsy. Five years after diagnosis one-fifth of patients still had at least one annual visit to a neurology department and 7.5\% had a hospital visit associated with epilepsy. Despite the increased neurological morbidity, overall rates of use of hospital services approached the rates seen in the comparison cohort following the first year of diagnosis. The annual number of sick leave days returned to the same level as in the comparison cohort after two years. Of note, many patients had stopped their professional work which may partly explain the return to average levels of sick leave days in the remaining patients capable of returning to work after their episode of HSV-1 CNS infection.

While HSV-2 CNS infection may be associated with recurrent meningitis and short-term neurological morbidity, ${ }^{8,9}$ our study corroborates the benign long-term prognosis of HSV-2 CNS infection, with little risk of major disability. The increased risk of death observed during the first year of follow-up was mainly attributable to an increased proportion of patients diagnosed with cancer before diagnosis of HSV-2 CNS infection. HSV-2 is more likely to be reactivated in immunocompromised patients, including patients receiving chemotherapy for malignant disorders and especially in patients receiving treatment for hematological malignancies. ${ }^{25}$ Further, immunocompromised patients have more serious HSV-2 manifestations. $^{26,27}$ These factors may explain the increased proportion with a cancer diagnosis prior to study inclusion in the HSV-2 cohort. In a previous Danish study of 49 patients 
A

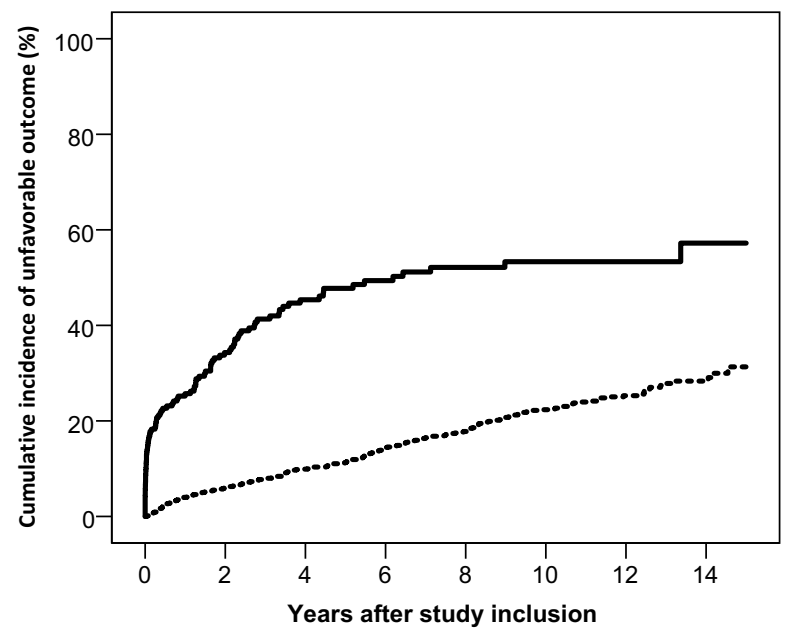

\section{B}

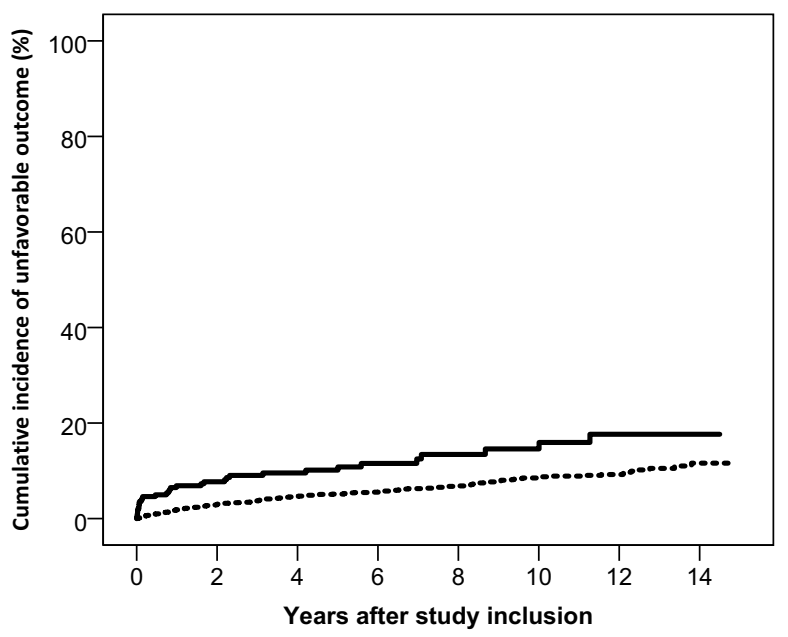

C

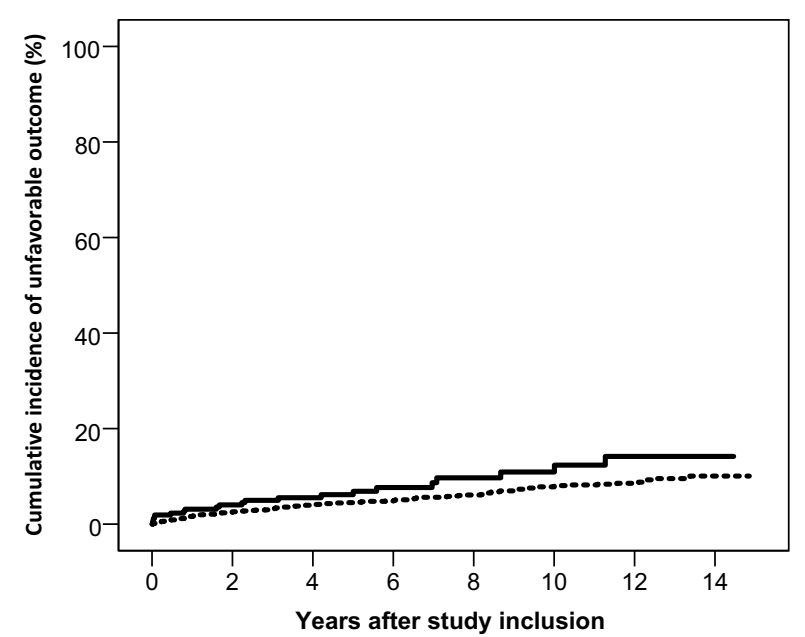

Figure 3 Cumulative incidence of an unfavorable outcome (death, dementia, receipt of a disability pension, and nursing home residency) in patients with herpes simplex virus (HSV) type I and type 2 central nervous system (CNS) infections (full lines) and members of the comparison cohorts (broken lines). (A) Unfavorable outcome after HSV-I CNS infection. (B) Unfavorable outcome after HSV-2 CNS infection. (C) Unfavorable outcome after HSV-2 CNS infection in persons without a prior diagnosis of cancer.

with HSV-2 CNS infection, there were no deaths resulting from CNS disease, but one patient died from malignant lymphoma. ${ }^{8}$

The HSV-2 patients in the current study had slightly increased use of hospital inpatient and outpatient services both before and after study inclusion. Our data does not allow us to explain this finding, but it is possible that symptoms from primary and recurrent genital HSV-2 $2^{28-30}$ lead to increased use of hospital services. In two other series of patients with HSV-2 CNS infection, $20 \%$ and $45 \%$ had more than one episode of meningitis, which also may have increased the use of health care services. ${ }^{8,10}$ Further, in our study HSV-2 patients had higher levels of comorbidity than the comparison cohort. Five years after diagnosis of the
HSV-2 CNS infection, the proportion of patients with at least one annual visit to a neurology department also remained around five percentage points higher than in the comparison cohort. However, the measures of increased morbidity did not translate into increased rates of unemployment or receipt of disability pensions. In contrast to HSV-1 patients, the risk of epilepsy was not persistently increased in the HSV-2 group except during the first year after diagnosis. It remains possible that some patients with convulsions, during the acute phase of HSV CNS infection, may have been misdiagnosed with epilepsy. Our results are in line with the results of a smaller single-center study, which found no neurological disability or death among 28 patients with HSV-2 meningitis during median follow-up time of 3.4 
years. ${ }^{10}$ In our study, the proportion divorced in the HSV-2 group was higher than in the comparison cohort before, as well as after, HSV-2 diagnosis. This may partly reflect that HSV-2 is a sexually transmitted disease associated with partner changes or greater numbers of sexual partners. ${ }^{31}$

\section{Conclusions}

In summary, our study demonstrates that HSV-1 and HSV-2 CNS infections have distinct long-term prognoses. HSV-1 CNS infection has high one-year mortality and survivors are at considerable risk of neurological sequelae and long-term disability. In contrast, HSV-2 CNS infection has no substantial impact on mortality or long-term risk of serious neurological sequelae and disability, although a diagnosis of HSV-2 CNS infection is associated with some measures of increased morbidity.

\section{Data Sharing Statement}

Data are stored in Statistics Denmark and according to Danish data protection cannot be shared.

\section{Funding}

The study was supported by the Danish Council for Independent Research, grant number: [6110-00173B]. The sponsor had no influence on the preparation, design, analysis, or reporting of this study.

\section{Disclosure}

RBD reports personal fees from Roche Diagnostics, outside the submitted work. A-ML reports personal fees from GSK, non-financial support from Gilead, outside the submitted work. HTS was supported by the PROCRIN program and also reports that The Department of Clinical Epidemiology, Aarhus University Hospital, receives funding for other studies from companies in the form of research grants to (and administered by) Aarhus University. None of these studies have any relation to the present study. The authors report no other conflicts of interest in this work.

\section{References}

1. Granerod J, Ambrose HE, Davies NW, et al. Causes of encephalitis and differences in their clinical presentations in England: a multicentre, population-based prospective study. Lancet Infect Dis. 2010;10(12):835-844. doi:10.1016/S1473-3099(10)70222-X

2. McGill F, Griffiths MJ, Bonnett LJ, et al. Incidence, aetiology, and sequelae of viral meningitis in UK adults: a multicentre prospective observational cohort study. Lancet Infect Dis. 2018;18(9):992-1003. doi:10.1016/S1473-3099(18)30245-7
3. Bodilsen J, Storgaard M, Larsen L, et al. Infectious meningitis and encephalitis in adults in Denmark: a prospective nationwide observational cohort study (DASGIB). Clin Microbiol Infect. 2018;24 (10):1102. doi: $10.1016 /$ j.cmi.2018.01.016

4. Jorgensen LK, Dalgaard LS, Ostergaard LJ, Norgaard M, Mogensen TH. Incidence and mortality of herpes simplex encephalitis in Denmark: a nationwide registry-based cohort study. $J$ Infect. 2017;74(1):42-49. doi:10.1016/j.jinf.2016.09.004

5. Moon SM, Kim T, Lee EM, Kang JK, Lee SA, Choi SH. Comparison of clinical manifestations, outcomes and cerebrospinal fluid findings between herpes simplex type 1 and type 2 central nervous system infections in adults. J Med Virol. 2014;86(10):1766-1771. doi:10.1002/ jmv.23999

6. Erdem H, Cag Y, Ozturk-Engin D, et al. Results of a multinational study suggest the need for rapid diagnosis and early antiviral treatment at the onset of herpetic meningoencephalitis. Antimicrob Agents Chemother. 2015;59(6):3084-3089. doi:10.1128/AAC.05016-14

7. Cooper J, Kierans C, Defres S, Easton A, Kneen R, Solomon T. Care beyond the hospital ward: understanding the socio-medical trajectory of herpes simplex virus encephalitis. BMC Health Serv Res. 2017;17 (1):646. doi:10.1186/s12913-017-2608-2

8. Omland LH, Vestergaard BF, Wandall JH. Herpes simplex virus type 2 infections of the central nervous system: a retrospective study of 49 patients. Scand J Infect Dis. 2008;40(1):59-62. doi:10.1080/ 00365540701509881

9. Aurelius E, Forsgren M, Gille E, Skoldenberg B. Neurologic morbidity after herpes simplex virus type 2 meningitis: a retrospective study of 40 patients. Scand J Infect Dis. 2002;34(4):278-283. doi:10.1080/00365540110080485

10. Miller S, Mateen FJ, Aksamit AJ Jr. Herpes simplex virus 2 meningitis: a retrospective cohort study. J Neurovirol. 2013;19(2):166-171. doi:10.1007/s13365-013-0158-x

11. Singh TD, Fugate JE, Hocker S, Wijdicks EFM, Aksamit AJ Jr., Rabinstein AA. Predictors of outcome in HSV encephalitis. J Neurol. 2016;263(2):277-289. doi:10.1007/s00415-015-7960-8

12. Hjalmarsson A, Blomqvist P, Skoldenberg B. Herpes simplex encephalitis in Sweden, 1990-2001: incidence, morbidity, and mortality. Clin Infect Dis. 2007;45(7):875-880. doi:10.1086/521262

13. Dagsdottir HM, Sigurethardottir B, Gottfreethsson M, et al. Herpes simplex encephalitis in Iceland 1987-2011. Springerplus. 2014;3:524. doi:10.1186/2193-1801-3-524

14. Jouan Y, Grammatico-Guillon L, Espitalier F, Cazals X, Francois P, Guillon A. Long-term outcome of severe herpes simplex encephalitis: a population-based observational study. Crit Care. 2015;19:345.

15. Sili U, Kaya A, Mert A. Herpes simplex virus encephalitis: clinical manifestations, diagnosis and outcome in 106 adult patients. J Clin Virol. 2014;60(2):112-118. doi:10.1016/j.jcv.2014.03.010

16. Hemingway H, Croft P, Perel P, et al. Prognosis research strategy (PROGRESS) 1: a framework for researching clinical outcomes. BMJ. 2013;346:e5595. doi:10.1136/bmj.e5595

17. Obel N, Dessau RB, Krogfelt KA, et al. Long term survival, health, social functioning, and education in patients with European Lyme neuroborreliosis: nationwide population based cohort study. BMJ. 2018;361:k1998. doi:10.1136/bmj.k1998

18. Roed C, Omland LH, Skinhoj P, Rothman KJ, Sorensen HT, Obel N. Educational achievement and economic self-sufficiency in adults after childhood bacterial meningitis. JAMA. 2013;309 (16):1714-1721. doi:10.1001/jama.2013.3792

19. Schmidt M, Schmidt SA, Adelborg K, et al. From healthcare contacts to database records. Clin Epidemiol. 2019;12(11):563-591. doi:10.2147/CLEP.S179083

20. Schmidt M, Pedersen L, Sorensen HT. The Danish Civil Registration System as a tool in epidemiology. Eur J Epidemiol. 2014;29 (8):541-549. doi:10.1007/s10654-014-9930-3 
21. Charlson ME, Pompei P, Ales KL, MacKenzie CR. A new method of classifying prognostic comorbidity in longitudinal studies: development and validation. $J$ Chronic Dis. 1987;40(5):373-383. doi:10.1016/0021-9681(87)90171-8

22. Thygesen SK, Christiansen CF, Christensen S, Lash TL, Sorensen HT. The predictive value of ICD-10 diagnostic coding used to assess Charlson comorbidity index conditions in the population-based Danish National Registry of Patients. BMC Med Res Methodol. 2011;11:83. doi:10.1186/1471-2288-11-83

23. Solomon T, Michael BD, Smith PE, et al. Management of suspected viral encephalitis in adults - Association of British Neurologists and British Infection Association National Guidelines. J Infect. 2012;64 (4):347-373. doi:10.1016/j.jinf.2011.11.014

24. Jorgensen LK, Dalgaard LS, Ostergaard LJ, Andersen NS, Norgaard M, Mogensen TH. Validity of the coding for herpes simplex encephalitis in the Danish National Patient Registry. Clin Epidemiol. 2016;8:133-140. doi:10.2147/CLEP.S104379

25. Taplitz RA, Kennedy EB, Bow EJ, et al. Antimicrobial prophylaxis for adult patients with cancer-related immunosuppression: ASCO and IDSA clinical practice guideline update. J Clin Oncol. 2018;36 (30):3043-3054. doi:10.1200/JCO.18.00374
26. Mateen FJ, Miller SA, Aksamit AJ Jr. Herpes simplex virus 2 encephalitis in adults. Mayo Clin Proc. 2014;89(2):274-275. doi:10.1016/j.mayocp.2013.12.003

27. Mommeja-Marin H, Lafaurie M, Scieux C, Galicier L, Oksenhendler E, Molina JM. Herpes simplex virus type 2 as a cause of severe meningitis in immunocompromised adults. Clin Infect Dis. 2003;37(11):1527-1533. doi:10.1086/379520

28. Benedetti J, Corey L, Ashley R. Recurrence rates in genital herpes after symptomatic first-episode infection. Ann Intern Med. 1994;121 (11):847-854. doi:10.7326/0003-4819-121-11-199412010-00004

29. Fisman DN, Lipsitch M, Hook EW, Goldie SJ. Projection of the future dimensions and costs of the genital herpes simplex type 2 epidemic in the United States. Sex Transm Dis. 2002;29 (10):608-622. doi:10.1097/00007435-200210000-00008

30. Johnston C, Corey L. Current concepts for genital herpes simplex virus infection: diagnostics and pathogenesis of genital tract shedding. Clin Microbiol Rev. 2016;29(1):149-161. doi:10.1128/CMR.00043-15

31. Kjaer SK, Engholm G, Teisen C, et al. Risk factors for cervical human papillomavirus and herpes simplex virus infections in Greenland and Denmark: a population-based study. Am J Epidemiol. 1990;131 (4):669-682. doi:10.1093/oxfordjournals.aje.a115551

\section{Publish your work in this journal}

Clinical Epidemiology is an international, peer-reviewed, open access, online journal focusing on disease and drug epidemiology, identification of risk factors and screening procedures to develop optimal preventative initiatives and programs. Specific topics include: diagnosis, prognosis, treatment, screening, prevention, risk factor modification,

Submit your manuscript here: https://www.dovepress.com/clinical-epidemiology-journal systematic reviews, risk \& safety of medical interventions, epidemiology \& biostatistical methods, and evaluation of guidelines, translational medicine, health policies \& economic evaluations. The manuscript management system is completely online and includes a very quick and fair peer-review system, which is all easy to use. 